\title{
Annalisa Bottacin, Una lettera inedita di Auguste Mignet a Vincenzo Salvagnoli, amico di Stendhal
}

\section{Annarosa Poli}

\section{(2) OpenEdition}

1 Journals

\section{Edizione digitale}

URL: http://journals.openedition.org/studifrancesi/36371

DOI: 10.4000/studifrancesi.36371

ISSN: 2421-5856

\section{Editore}

Rosenberg \& Sellier

\section{Edizione cartacea}

Data di pubblicazione: 1 juillet 2005

Paginazione: 182

ISSN: 0039-2944

\section{Notizia bibliografica digitale}

Annarosa Poli, «Annalisa Bottacin, Una lettera inedita di Auguste Mignet a Vincenzo Salvagnoli, amico di Stendhal», Studi Francesi [Online], 145 (XLIX | I) | 2005, online dal 30 novembre 2015, consultato il 18 avril 2021. URL: http://journals.openedition.org/studifrancesi/36371 ; DOI: https://doi.org/10.4000/ studifrancesi.36371

\section{Questo documento è stato generato automaticamente il 18 avril 2021.}

\section{(c) (1)}

Studi Francesi è distribuita con Licenza Creative Commons Attribuzione - Non commerciale - Non opere derivate 4.0 Internazionale. 


\title{
Annalisa Bottacin, Una lettera inedita di Auguste Mignet a Vincenzo Salvagnoli, amico di Stendhal
}

\author{
Annarosa Poli
}

\section{NOTIZIA}

ANNALISA BOTTACIN, Una lettera inedita di Auguste Mignet a Vincenzo Salvagnoli, amico di Stendhal, «Quaderni del C.R.I.E.R», n 5, 2002, pp. 61-73.

1 L'ultimo viaggio che portò Stendhal da Civitavecchia a Parigi risale all'autunno del 1841; gli fu compagno un'importante figura dell'Italia preunitaria, l'amico fiorentino Vincenzo Salvagnoli, celebre giurista, uomo politico, propugnatore della libertà e dell'indipendenza, legato al Mazzini, di cui fu corrispondente negli stati toscani. Svariate ragioni sono alla base di questo périple che vide i due amici insieme in episodi di vita quotidiana. La mèta designata prevedeva infatti una sosta a Ginevra dove Henri Beyle, in cattive condizioni fisiche, doveva consultare un eminente clinico, che già in epoche precedenti lo aveva curato: Jean-Louis Prévost. Nella città svizzera, che gli aveva dato i natali, Stendhal e Salvagnoli avrebbero anche incontrato Abraham Constantin, l'artista che divise con lo scrittore alcuni alloggi romani, autore delle Idées italiennes sur quelques tableaux célèbres, un volume pubblicato nel 1840 da Giovan Pietro Vieusseux, di cui certo Stendhal non corresse solo le bozze, bensì ebbe una parte integrante nella redazione.

Giunto nella capitale francese, Salvagnoli fu introdotto dall'autore del Rouge nei salotti parigini frequentati dalla sinistra liberale; ebbe tra l'altro occasione di incontrare figure di spicco quali Cousin, Thiers, Constant e Mignet. È con quest'ultimo, che protesse in più di un'occasione Henri Beyle durante gli anni consolari, che l'avvocato empolese si legò di una sincera amicizia. In effetti, nel Fondo Salvagnoli Marchetti, proveniente dalla villa di famiglia di Corniola ed ora depositato presso l'Archivio 
Storico Comunale di Empoli, che Annalisa Bottacin ha reperito una lettera autografa di Auguste Mignet, inviata a Vincenzo Salvagnoli, datata 22 dicembre 1843, che fu recapitata da Giuseppe Massari, giovane patriota che rientrava da Parigi a Firenze. La missiva, scritta dunque un anno dopo il rientro in Italia dello statista, oltre a rinnovare il rapporto di stima ed amicizia, reca un post-scriptum in cui Mignet chiede a Salvagnoli una ricerca intorno ad alcuni documenti relativi ai senesi fratelli Socini, fautori del Socinianesimo, corrente teologica radicale in seno alla riforma protestante del XVI secolo.

In effetti, Stendhal nei suoi contributi alla stampa inglese tratta sovente di Mignet e dei suoi scritti e più di una volta accenna a un progetto che avrebbe occupato per molti anni Mignet, relativo ad una Histoire de la Réformation de la Ligue et du règne de Henri IV; opera che dal 1826 fu varie volte annunciata anche da Quérard, ma che per altro non fu mai edita. Nelle carte salvagnoliane del medesimo Fondo, è stata anche reperita una carta, redatta probabilmente da un segretario, che riporta di mano di Salvagnoli il mittente: "A $\mathrm{M}^{\mathrm{r}}$ Mignet, 12 avril" con omissione dell'anno, che tuttavia non può considerarsi una risposta alla missiva precedente, pur attestando la prosecuzione di un rapporto tra i due storici, entrambi legati al comune amico grenoblese. 